\title{
Reduced plasma levels of glucagon-like peptide-1 in elderly men are associated with impaired glucose tolerance but not with coronary heart disease
}

\author{
D. Nathanson • B. Zethelius • C. Berne • J. J. Holst • \\ Å. Sjöholm • T. Nyström
}

Received: 1 September 2009 /Accepted: 6 October 2009/Published online: 21 November 2009

(C) Springer-Verlag 2009

\begin{abstract}
Aims/hypothesis Besides the insulinotropic effects of glucagon-like peptide-1 (GLP-1) mimetics, their effects on endothelial dysfunction and myocardial ischaemia are of interest. No previous study has investigated associations between plasma levels of GLP-1 and CHD.

Methods We investigated longitudinal relationships of fasting GLP-1 with the dynamic GLP-1 response after OGTT (difference between 60 min OGTT-stimulated and fasting GLP-1 levels $[\Delta \mathrm{GLP}-1])$ and CHD in a populationbased cohort of 71-year-old men. In the same cohort, we also cross-sectionally investigated the association between stimulated GLP-1 levels and: (1) cardiovascular risk factors (blood pressure, lipids, urinary albumin, waist circumference and insulin sensitivity index $[M / I]$ assessed by euglycaemic-hyperinsulinaemic clamp); and (2) impaired glucose tolerance (IGT) and type 2 diabetes mellitus.
\end{abstract}

\footnotetext{
D. Nathanson $(\varangle) \cdot \AA$. Sjöholm $\cdot$ T. Nyström

Department of Internal Medicine, Karolinska Institutet,

Södersjukhuset AB,

SE-118 83 Stockholm, Sweden

e-mail: david.nathanson@sodersjukhuset.se

B. Zethelius

Department of Public Health and Caring Sciences/Geriatrics,

Uppsala University,

Uppsala, Sweden

C. Berne

Department of Medical Sciences, Uppsala University Hospital,

Uppsala, Sweden

J. J. Holst

Department of Biomedical Sciences, The Panum Institute,

University of Copenhagen,

Copenhagen, Denmark
}

Results During the follow-up period (maximum 13.8 years), of 294 participants with normal glucose tolerance (NGT), 69 experienced a CHD event (13.8 years), as did 42 of 141 with IGT and 32 of 74 with type 2 diabetes mellitus. $\triangle$ GLP-1 did not predict CHD (HR 1.0, 95\% CI 0.52-2.28). The prevalence of IGT was associated with $\Delta$ GLP-1, lowest vs highest quartile (OR $0.3,95 \%$ CI $0.12-0.58$ ), with no such association for type 2 diabetes mellitus (OR 1.0, 95\% CI 0.38-2.86). M/I was significantly associated with $\triangle \mathrm{GLP}-1$ in the type 2 diabetes mellitus group $(r=0.38, p<0.01)$, but not in the IGT $(r=0.11, p=0.28)$ or NGT $(r=0.10, p=0.16)$ groups.

Conclusions/interpretation Impaired GLP-1 secretion is associated with IGT, but not with type 2 diabetes mellitus. This finding in the latter group might be confounded by oral glucose-lowering treatment. GLP-1 does not predict CHD. Although $\triangle$ GLP-1 was associated with insulin sensitivity in the type 2 diabetes mellitus group, GLP-1 does not seem to be a predictor of CHD in insulin-resistant patients.

Keywords Coronary heart disease $\cdot$ Glucagon-like peptide- $1 \cdot$ Impaired glucose tolerance $\cdot$ Type 2 diabetes

\author{
Abbreviations \\ GLP-1 Glucagon-like peptide-1 \\ $\Delta$ GLP-1 Difference between 60 min OGTT-stimulated \\ and fasting GLP-1 levels \\ IGT Impaired glucose tolerance \\ $M / \mathrm{I} \quad$ Insulin sensitivity index \\ NGT Normal glucose tolerance
}

\section{Introduction}

Besides the documented insulinotropic effects of glucagonlike peptide-1 (GLP-1) mimetics, their effects on endothe- 
lial dysfunction and myocardial ischaemia are of great interest [1]. Cohort studies have shown a slightly reduced meal-stimulated response in GLP-1 secretion in type 2 diabetic patients and in participants with impaired glucose tolerance (IGT) [2]. We wanted to investigate whether plasma levels of GLP-1 after an OGTT predict CHD in a community-based cohort of elderly men. A further aim was to investigate cross-sectionally in the same cohort whether plasma levels of GLP-1 are associated with: (1) risk factors for CHD, i.e. blood pressure, lipids, urinary albumin, waist circumference and insulin sensitivity; and (2) IGT and type 2 diabetes mellitus.

\section{Methods}

Study population Random plasma samples from 509 men, obtained from the previously described ULSAM cohort study (www.pubcare.uu.se/ULSAM, accessed 2 September 2009) [3], were stored at $-70^{\circ} \mathrm{C}$ and analysed in 2007. In the prospective analyses, 62 men were excluded due to prior myocardial infarction or angina pectoris (ICD-9 codes 410-414, ICD-10 codes I20-25 [www.who.int/classifica tions/icd/en/, accessed 1 October 2007]). At baseline, participants' medical histories and current pharmacological treatments were collected using the original protocol questionnaires. CHD mortality and morbidity rates were collected from The Center for Epidemiology, National Board of Health and Welfare, Sweden. The study was approved by the Ethics Committee of the Faculty of Medicine at Uppsala University. Written informed consent was obtained from all participants.

Baseline characteristics Blood samples were drawn in the morning after an overnight fast and had been stored at $-70^{\circ} \mathrm{C}$ since baseline. Plasma concentrations of GLP-1 were measured in single samples by radio-immunoassay [4]. Type 2 diabetes mellitus and IGT were defined according to the WHO 1999 criteria using a 75 g OGTT or by the use of oral hypoglycaemic agents. Fasting concentrations of plasma glucose and serum lipids, waist circumference, an electrocardiogram, and supine systolic and diastolic blood pressure were measured under standardised conditions [5].

Follow-up data CHD was defined either on the basis of cause of death as recorded in the Cause of Death Registry, or in patients first hospitalised for CHD on the basis of diagnoses (ICD-9 codes 410-414, ICD-10 codes I20-25) recorded in the Hospital Discharge Registry (censor date December 31, 2006). CHD morbidity, defined by combining data from the Cause of Death Registry and the Hospital Discharge Registry, is an efficient and validated alternative to revised hospital notes and death certificates [6]. No participant was lost to follow-up due to missing registry data.

OGTT and euglycaemic-hyperinsulinaemic clamp Glucose tolerance was assessed by a $75 \mathrm{~g}$ oral OGTT. Insulin sensitivity index $(M / \mathrm{I})$ was determined with the euglycaemic-hyperinsulinaemic clamp technique [3, 7]. Plasma glucose was analysed by a device using glucose dehydrogenase method (Gluc-DH; Merck, Darmstadt, Germany).

Statistical analyses Logarithmic transformation was performed to achieve normal distribution if necessary. Tests were two-tailed, with a $p$ value of less than 0.05 deemed statistically significant. We created a dynamic predicted variable by calculating the difference between $60 \mathrm{~min}$ OGTT-stimulated and fasting GLP-1 levels ( $\Delta$ GLP-1) as follows: 60 min GLP-1 - fasting GLP-1 = $\Delta$ GLP-1. Cox's proportional hazard regression analyses were used in the prospective analyses. Participants were divided into quartiles according to their $\triangle$ GLP-1 levels. For participants experiencing a CHD event, time from baseline to event in the different groups of quartiles was compared by logrank test and results presented as Kaplan-Meier curves. Logistic regression analysis was used to study associations between the GLP-1 variables and dichotomous dependent variables. Potential associations were presented as OR $(95 \% \mathrm{CI})$ and tested with the Wald test. For IGT and type 2 diabetes mellitus, adjustments were made for $M / \mathrm{I}$, plasma insulin and waist circumference. Adjustments in analysis of CHD were made for BMI, systolic blood pressure, urinary albumin and lipids. Pearson's correlation analysis was performed between $\triangle$ GLP-1 and risk factors for CHD. Differences in $\triangle$ GLP-1 between the groups were assessed by ANOVA one-way repeated measurement, followed by Bonferroni's post-hoc test. All statistical analyses were performed using the statistical software package SPSS 17.0 for PC (SPSS, Chicago, IL, USA).

\section{Results}

Patient characteristics Baseline clinical characteristics are presented in Table 1. No differences in fasting GLP-1 levels were seen between groups. $\triangle$ GLP-1 was significantly lower in the IGT group than in the groups with normal glucose tolerance (NGT) and type 2 diabetes mellitus, respectively (Table 1).

Cross-sectional outcome A high $\Delta$ GLP-1 (lowest vs highest quartile) was associated with lower risk of IGT (OR 0.3, 95\% CI $0.12-0.58$ ), with no association in the type 2 diabetes mellitus group (OR 1.0, 95\% CI 0.38-2.86). No association was demonstrated between fasting GLP-1 (OR 1.4, 95\% CI 
Table 1 Baseline clinical and biochemical characteristics of the total study sample and the three groups according to glucose tolerance at age 71 years

\begin{tabular}{|c|c|c|c|c|}
\hline Characteristic & Total $(n=509)$ & NGT $(n=294)$ & $\operatorname{IGT}(n=141)$ & Type 2 diabetes $(n=74)$ \\
\hline Fasting plasma glucose (mmol/l) & $5.8 \pm 0.1$ & $5.3 \pm 0.1$ & $5.6 \pm 0.1^{*}$ & $8.1 \pm 0.3^{* * \dagger}$ \\
\hline $120 \mathrm{~min}$ plasma glucose $(\mathrm{mmol} / \mathrm{l})$ & $8.4 \pm 0.2$ & $6.0 \pm 0.1$ & $9.1 \pm 0.1^{*}$ & $16.8 \pm 0.5^{* * \dagger}$ \\
\hline Fasting GLP-1 $(\mathrm{pmol} / \mathrm{l})^{\mathrm{a}}$ & $11.7 \pm 0.7$ & $10.3 \pm 0.8$ & $13.6 \pm 1.5$ & $13.4 \pm 2.0$ \\
\hline $60 \min$ GLP-1 $(\mathrm{pmol} / 1)^{\mathrm{a}}$ & $18.7 \pm 1.1$ & $19.8 \pm 1.5$ & $15.9 \pm 1.7$ & $19.3 \pm 2.3$ \\
\hline$\Delta$ GLP-1 $(\mathrm{pmol} / \mathrm{l})$ & $7.9 \pm 1.0$ & $10.1 \pm 1.5$ & $3.2 \pm 1.2 *$ & $7.9 \pm 1.9$ \\
\hline Fasting insulin $(\mathrm{pmol} / \mathrm{l})^{\mathrm{a}}$ & $85.2 \pm 2.4$ & $76.8 \pm 2.4$ & $89.4 \pm 3.6$ & $109.8 \pm 12.0^{* *}$ \\
\hline $60 \min$ insulin $(\mathrm{pmol} / \mathrm{l})^{\mathrm{a}}$ & $455.4 \pm 13.2$ & $448.8 \pm 18.0$ & $469.2 \pm 25.8$ & $449.4 \pm 30.6$ \\
\hline$\Delta$ Insulin $(\mathrm{pmol} / \mathrm{l})$ & $369 \pm 14.4$ & $372.6 \pm 18.0$ & $379.2 \pm 26.4$ & $337.8 \pm 33.0$ \\
\hline$M / \mathrm{I}\left(\mathrm{mg} \min ^{-1} \mathrm{~kg}^{-1} /[100 \mathrm{mU} / 1]\right)^{\mathrm{b}}$ & $4.9 \pm 0.1$ & $5.7 \pm 1.3$ & $4.3 \pm 0.2^{*}$ & $2.8 \pm 0.2 * *, \dagger$ \\
\hline Total cholesterol (mmol/l) & $5.8 \pm 0.1$ & $5.9 \pm 0.1$ & $5.8 \pm 0.1$ & $5.7 \pm 0.1$ \\
\hline LDL-cholesterol (mmol/l) & $3.9 \pm 0.1$ & $4.0 \pm 0.1$ & $3.9 \pm 0.1$ & $3.8 \pm 0.1$ \\
\hline Triacylglycerol (mmol/l) & $1.4 \pm 0.1$ & $1.3 \pm 0.1$ & $1.5 \pm 0.1^{*}$ & $1.7 \pm 0.1 * *$ \\
\hline Systolic BP (mmHg) & $145 \pm 0.8$ & $143 \pm 1.1$ & $147 \pm 1.6$ & $152 \pm 2.3 * *$ \\
\hline Diastolic BP (mmHg) & $82 \pm 0.4$ & $81 \pm 0.5$ & $83 \pm 0.9$ & $84 \pm 1.2$ \\
\hline Urinary albumin $(\mu \mathrm{g} / \mathrm{min})$ & $24.6 \pm 3.9$ & $23.2 \pm 5.2$ & $16.6 \pm 4.0$ & $44.9 \pm 15.1$ \\
\hline Waist circumference $(\mathrm{cm})$ & $94 \pm 0.4$ & $93 \pm 0.5$ & $94 \pm 0.7 *$ & $98 \pm 1.1^{* *}$ \\
\hline
\end{tabular}

Data are presented as means \pm SEM

${ }^{a}$ The difference between 60 min glucose-stimulated GLP-1 and fasting GLP-1 is expressed as $\Delta$ GLP-1 and similarly also for insulin

${ }^{\mathrm{b}} \mathrm{M} / \mathrm{I}$ was calculated as glucose disposal rate (infused glucose in $\mathrm{mg} \mathrm{min}^{-1}$ [body weight $]^{-1}$ ) divided by mean plasma insulin concentration $(100 \mathrm{mU} / \mathrm{l})$ during the last $60 \mathrm{~min}$ of the $2 \mathrm{~h}$ euglycaemic-hyperinsulinaemic clamp. To convert values to SI units ( $\left.\mathrm{mg} \mathrm{min}^{-1} \mathrm{~kg}^{-1} /[695 \mathrm{pmol} / 1]\right)$, divide values by 6.95

${ }^{*} p<0.05,{ }^{* *} p<0.01$ vs NGT group; ${ }^{\dagger} p<0.05$ vs IGT group (one-way repeated measurement [ANOVA])

0.78-2.53) or 60 min GLP-1 (OR 0.82, 95\% CI 0.42-1.6) for the presence of IGT. There were no associations between GLP-1 and fasting or post-load glucose levels in the IGT and type 2 diabetes mellitus, groups (data not shown). In contrast, a significant association was noted between $\Delta$ GLP-1 and $M / \mathrm{I}$ in the type 2 diabetes mellitus group $(r=0.38, p<0.01)$, with no such associations discerned in the IGT $(r=0.11, p=0.28)$ or NGT $(r=0.10, p=0.16)$ groups. There were no associations between GLP-1 (fasting GLP-1, 60 min GLP-1 or $\Delta$ GLP-1) levels and other known risk markers for CHD.

Follow-up outcome During follow-up, 69 of 294 NGT participants (rate 2.6/100 person-years at risk) experienced a CHD event, as did 42 of 141 IGT and 32 of 74 type 2 diabetes mellitus participants (rate 3.5/100 and 6.0/100 person-years at risk respectively). The median follow-up time was 12.0 years (range 0.2 to 13.8 ) with a total of 4,396 person-years at risk. There was no association between the lowest vs the highest quartile of $\triangle$ GLP-1 and CHD, based on 143 individuals who developed CHD (HR 1.0, 95\% CI 0.52-2.28). Unadjusted Kaplan-Meier survival curves failed to demonstrate any association between quartiles of $\triangle$ GLP-1 and risk of CHD (Fig. 1). During the same follow-up period, neither fasting GLP-1 (HR 1.03, 95\% CI 0.5-2.1), nor 60 min GLP-1 (HR 0.79, 95\% CI 0.37-1.6) correlated with CHD.

\section{Discussion}

In the current study, elderly men who are free from CHD at age 71 have a 2.3-fold elevated CHD risk if they have type 2 diabetes mellitus. Our primary aim, to investigate whether plasma levels of GLP-1 are associated with this high incidence of $\mathrm{CHD}$, was not proven. Neither were any

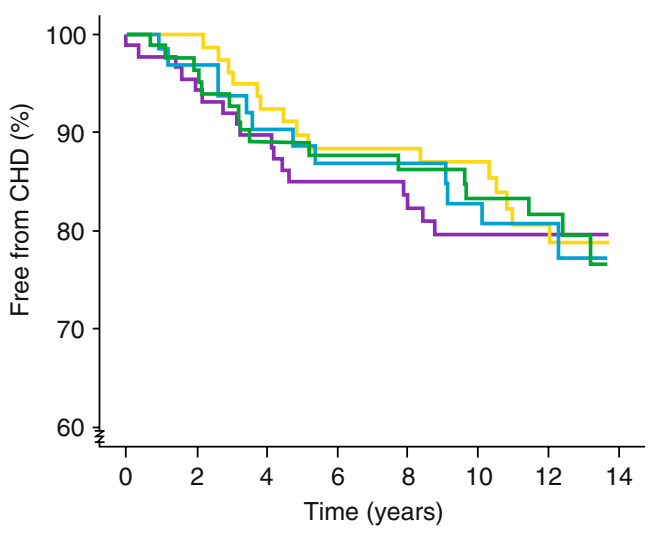

Fig. 1 Kaplan-Meier survival curves for quartiles of $\triangle$ GLP-1 in participants free from subsequent CHD events during 13.8 years of follow-up. No association was seen between the order of quartile (blue line, first quartile; green line, second quartile; yellow line, third quartile; purple line, fourth quartile) and both risk of CHD and $\triangle \mathrm{GLP}-1$ 
associations observed between risk factors for CHD and plasma levels of GLP-1, with the exception of $M / \mathrm{I}$, where a positive correlation between $\Delta$ GLP-1 and $M /$ I was noted, but only in the type 2 diabetes mellitus group. As expected, $M /$ I was significantly lower in the type 2 diabetes mellitus group than in the other groups, indicative of insulin resistance in this group. The reason for the lack of association between $\Delta \mathrm{GLP}-1$ and $M / \mathrm{I}$ in the other groups remains elusive.

GLP-1 might suppress hepatic glucose production directly [8] or indirectly by regulating glucagon secretion, which controls hepatic glucose production. The euglycaemic-hyperinsulinaemic clamp, used in the present study without infusion of radioactive-marked-glucose, cannot distinguish between hepatic vs skeletal muscle insulin sensitivity. We thus cannot rule out that hepatic glucose production was different between groups during the euglycaemic-hyperinsulinaemic clamp.

The incapacity to increase GLP-1 levels after an OGTT, as seen in IGT participants, was not apparent in the type 2 diabetes mellitus group, which had a similar response to that of NGT participants. The reason for this discrepancy is not clear. However, it might be explained by the oral hypoglycaemic agents used in the type 2 diabetes mellitus group. Almost one third of the type 2 diabetes mellitus group was taking oral hypoglycaemic agents, predominantly metformin. Metformin increases GLP-1 plasma levels by a similar magnitude to that produced by dipeptidyl peptidase inhibitors, probably due to inhibition of GLP-1 degradation [9]. Therefore, the increased GLP-1 levels in the type 2 diabetes mellitus group might be explained by metformin treatment.

Several limitations of this study need to be addressed. One reason for the lack of association between GLP-1 levels and CHD could be a lack of power, if a possible effect of GLP-1 is smaller than can be observed. Also, the population of 71-year-old men without CHD at baseline might have been too narrowly selected. In addition, plasma levels of GLP-1 were analysed in samples that had been frozen for more than 10 years (albeit at $-70^{\circ} \mathrm{C}$ ) and this might have influenced peptide stability. However, in unpublished observations we have noted that GLP-1 is stable over several years when kept frozen (J.J. Holst, unpublished data). Also, some data on the long storage of peptides, e.g. proinsulin, in this same cohort demonstrate that no significant degradation occurred over 25 years of storage time [3]. Even if a minor degradation cannot be ruled out, it should have proceeded at the same rate in all samples and so its impact on the outcome and interpretation of the data can be assumed to be minor. We have no obvious explanation as to why some individuals demonstrated negative $\Delta$ GLP-1 after OGTT. While this might be a true biological response in certain individuals, we cannot rule out the possibility that GLP-1 levels after OGTT might have peaked at another time. In some participants, $\Delta$ GLP-1 values were highly negative $(<25 \mathrm{pmol} / \mathrm{l})$. Nonetheless, after adjusting for these outliers, the same results remain (somewhat less pronounced, but still statistically significant).

Notwithstanding these potential caveats, plasma levels of GLP-1, at least in this cohort study of elderly men, is not a predictor of CHD.

Acknowledgements We thank H. Pettersson (Department of Biostatistics at Department of Clinical Science and Education, Södersjukhuset, Karolinska Institutet, Stockholm) and M. Nermo (Swedish Institute for Social Research, Stockholm University) for excellent statistical advice. Financial support was provided through the regional agreement on medical training and clinical research (ALF) between Stockholm County Council and the Karolinska Institute, and by the Swedish Society for Medical Research, the Swedish Society of Medicine, Stiftelsen Serafimerlasarettet, the European Foundation for the Study of Diabetes, Karolinska Institute Foundations, the Åke Wiberg fund and Stiftelsen Olle Engkvist Byggmästare.

Duality of interest The authors declare that there is no duality of interest associated with this manuscript.

\section{References}

1. Nyström T (2008) The potential beneficial role of glucagon-like peptide-1 in endothelial dysfunction and heart failure associated with insulin resistance. Horm Metab Res 40:593-606

2. Toft-Nielsen MB, Damholt MB, Madsbad S et al (2001) Determinants of the impaired secretion of glucagon-like peptide- 1 in type 2 diabetic patients. J Clin Endocrinol Metab 86:3717-3723

3. Zethelius B, Byberg L, Hales CN, Lithell H, Berne C (2002) Proinsulin is an independent predictor of coronary heart disease: report from a 27-year follow-up study. Circulation 105:2153-2158

4. Nyström T, Gutniak MK, Zhang Q et al (2004) Effects of glucagonlike peptide- 1 on endothelial function in type 2 diabetes patients with stable coronary artery disease. Am J Physiol 287:E1209E1215

5. Byberg L, McKeigue PM, Zethelius B, Lithell HO (2000) Birth weight and the insulin resistance syndrome: association of low birth weight with truncal obesity and raised plasminogen activator inhibitor-1 but not with abdominal obesity or plasma lipid disturbances. Diabetologia 43:54-60

6. Merlo J, Lindblad U, Pessah-Rasmussen H et al (2000) Comparison of different procedures to identify probable cases of myocardial infarction and stroke in two Swedish prospective cohort studies using local and national routine registers. Eur J Epidemiol 16:235-243

7. DeFronzo RA, Tobin JD, Andres R (1979) Glucose clamp technique: a method for quantifying insulin secretion and resistance. Am J Physiol 237:E214-E223

8. Prigeon RL, Quddusi S, Paty B, D'Alessio DA (2003) Suppression of glucose production by GLP-1 independent of islet hormones: a novel extrapancreatic effect. Am J Physiol 285:E701-E707

9. Mannucci E, Ognibene A, Cremasco F et al (2001) Effect of metformin on glucagon-like peptide 1 (GLP-1) and leptin levels in obese nondiabetic subjects. Diabetes Care 24:489-494 